\title{
A NOVEL APPROACH TO OPTIMIZE THE RIDESHARING PROBLEM USING GENETIC ALGORITHM
}

\author{
S. Rangriz ${ }^{1, *}$, M. Davoodi ${ }^{2}$, J. Saberian $^{3}$ \\ ${ }^{1}$ M.Sc. Student in GIS at Department of Surveying Engineering, Technical and Engineering Faculty, Islamic AZAD University \\ South Tehran Branch, Iran - rangrizsamira@gmail.com \\ ${ }^{2}$ M.Sc. in GIS at Faculty of Geodesy and Geomatics, K.N.Toosi University of Technology, Iran - mojtaba.davoodi@ut.ac.ir \\ ${ }^{3}$ Technical and Engineering Faculty, Islamic AZAD University South Tehran Branch, Iran - \\ j_saberian@azad.ac.ir
}

Commission VI, WG VI/4

KEY WORDS: Ridesharing, Urban Traffic Management, Route Finding, Genetic Algorithm, Dijkstra

\begin{abstract}
:
The enormous increase in the number of vehicles in the cities makes plenty of problems including air pollution, noise pollution, and traffic jam. Overcoming these annoying issues needs a significant plan in urban management such as using modern techniques in public transportation systems. Sharing either cars or taxies is one of the most interesting ways that has been used in some countries recently. In this phenomenon, 2 or 3 people use other's car or taxi. In this article, an innovative approach to share taxies is proposed, and it uses a Genetic Algorithm to determine the placement of travelers in taxies. Therefore, some taxis will be switched off, and this helps to decrease urban traffic jam in cities. The results present that the proposed model turns off $69.8 \%$ of taxies, and also $27.8 \%$ of them carry more than one passenger; hence, this confirms the performance of the proposed model.
\end{abstract}

\section{INTRODUCTION}

Traffic and air pollution are two significant metropolitan problems in the country, and cars are the main reason for these problems. Traffic management is the first step in the elimination of metropolitan cities from traffic congestion and air pollution. Recently, experts in the field of transport have introduced "Shared Economy" to address many of these problems (Böckmann, 2013). This concept is sharing the assets of individuals between themselves, in such a way that each individual provides their immediate needs through the temporary possession of the assets of others. One of the issues in the sharing economy is Carpooling.

Carpooling is a kind of car-sharing so that more than one person can use a car simultaneously, and it is one of the best ways to reduce the number of cars in the cities (Charles and Kline, 2006). The benefits of carpooling are the elimination of tampering, saving fuel consumption, reducing traffic jams, lowering vehicle lateral costs, decreasing fuel consumption, reducing driving stress, and declining the need for parking. This saves the costs of both the car owner and passengers.

\section{LITERATURE REVIEW}

Wei et al solved routing for taxi-pooling problem based by using Ant Colony Optimization (ACO) algorithm (Davoodi and Mesgari, 2015). Three main aims are considered in this article: 1) minimizing the cost of passengers, 2) maximizing the benefit of drivers and 3) maximizing the load rate. The simulated results indicate that the developed method can effectively solve the taxi-pooling routing problem with the above three objectives (Wei et al., 2016).

Huang et al solved the carpooling problem by using Tabu Search algorithm. In this paper, a new model is proposed which involves several origins and one destination. This work is carried out by considering environmental pollution, parking problems, traffic jams, and low utilization of resources. A Tabu search algorithm is used to solve the problem, and it aims at a wide range of passenger distribution and routing problems. To evaluate the performance of the method, real data are used, and the empirical results prove the effectiveness of the developed algorithm (Huang et al., 2016).

Zhan et al used an Artificial Bee Colony algorithm for optimization of dynamic taxi sharing problem. The model proposes a mobile application platform which maximizes the total benefits of the taxi sharing network considering time, cost, and capacity constraints. Dividing the problem into many small continuous static sub-problems based on a specific time interval is the strategy of authors to solve it. The developed model is compared with the greedy randomized adaptive search procedure (GRASP) with path relinking proposed by Santos and Xavier (Santos and Xavier, 2015), and the results depict that the performance of the proposed ABC model is better than the GRASP model, and the average saving ratio can reach $26 \%$ at the cost of only $16 \%$ extra travel time (Zhan et al., 2019).

Huang et al used an ant path-oriented carpooling allocation strategy to optimize the carpool service obstacle with time windows. In this study, three methods were examined: 1) assignment-based ant colony optimization, 2) genetic algorithm, and 3) simulated annealing. These techniques are compared with path-based and assignment-based representations by examining two objective functions: 1) The main objective that maximizes the entire quantity of matched travelers and seat usage rates (SURs), and 2) a secondary one that descends passengers' distances (Huang et al., 2018).

\section{METHODOLOGY}

In this paper, firstly, the required data are generated and compiled. These data include the urban road network as a platform for calculations, passenger, and taxi information. The traveler's information includes the origin of the trip, the travel 
destination, the time of travel request, and the time limit for the trip's subscription. The origin point and capacity is also considered as taxies information. The simulation environment in this article is the urban road network, and passengers, taxies and control center (which performs calculations) is considered as agents that interact with each other, and make decisions.

In this paper, the focus is on the method of sorting and optimizing the travels, so the Dijkstra algorithm is used for routing. This algorithm is a kind of mathematical algorithms that find the definite answer to the problem (Dijkstra, 1959).

But in the main part of the problem, which is finding the best order for travelers, a genetic algorithm, which is one of the most widely used algorithms, is used. The method of making the answer (chromosome) in the genetic algorithm in this study is that a matrix which is constructed according to the number of taxies, and each row contains the index of different passengers indicating the arrangement of passenger's origins and destinations. The order of the origin and destination of passengers is determined randomly in each row for each taxi. The fitness function for each answer is the total distances between passengers in each taxi, and this total travel length is calculated for all taxies so that the total travel cost of each answer will be arranged in descending order. The scheme of a chromosome is shown in Figure 1.

\begin{tabular}{|l|l|l|l|l|l|l|l|l|}
\hline Chromosome structure: & $O_{x}$ & $O_{z}$ & $O_{w}$ & $D_{z}$ & $D_{w}$ & $O_{y}$ & $D_{x}$ & $D_{y}$ \\
\hline
\end{tabular}

Figure 1. The structure of genes including origins and destinations of passengers

As this problem solves the travel of passengers, the parameter that evaluates the problem is distance; consequently, the total length between origins and destinations of all passengers determines the fitness function. The total travelled distance of each car is:

$$
D=\sum_{i=1}^{n} d_{i}, n=1,2, \ldots, 7
$$

Where $\mathrm{n}$ is the number of distances between origin and destination of passengers, and when the maximum movements of a car are 8, the maximum distances between them is 7 .

All taxi arrangements are put together, and the answer is created. The final scheme of a chromosome (answer) is shown below.

\begin{tabular}{|c|c|c|c|c|c|c|c|c|}
\hline $1^{\text {st }}$ taxi & $O_{x}$ & $O_{z}$ & $O_{w}$ & $D_{z}$ & $D_{w}$ & $O_{y}$ & $D_{x}$ & $D_{y}$ \\
\hline $2^{\text {nd }}$ taxi & $O_{p}$ & $O_{f}$ & $D_{p}$ & $O_{h}$ & $O_{i}$ & $D_{h}$ & $D_{p}$ & $D_{i}$ \\
\hline$\cdots$ & & & & & & & & \\
\hline $\mathrm{n}^{\text {th }}$ taxi & $O_{t}$ & $D_{t}$ & $O_{b}$ & $O_{g}$ & $O_{k}$ & $D_{b}$ & $D_{b}$ & $D_{k}$ \\
\hline
\end{tabular}

Figure 2. The final structure of a chromosome (answer of problem)

Then, the total travel length of an answer is calculated by:

$$
L=\sum_{j=1}^{m} D_{j}
$$

The objective of the problem is minimizing the $\mathrm{L}$.

At this step, the origin-destination cost matrix must be created between all these points (origins and destinations of passengers and taxies). The OD matrix determines the network cost (Van Zuylen and Willumsen, 1980). For example, the route from one city to another may have a cost of 45 kilometers. The cost of the network can be anything but generally, it is the distance or time. A network data set must have at least one cost attribute for using in network analysis, because network analytics always optimize the cost.
Spatial Interactions

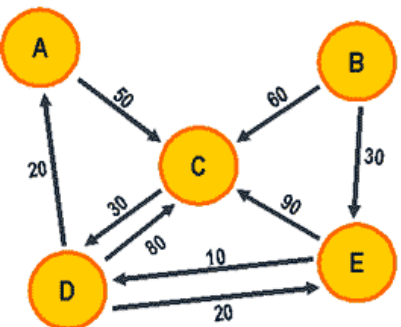

OID Matrix

\begin{tabular}{|c|c|c|c|c|c|c|}
\hline & A & B & C & D & E & T \\
\hline A & 0 & 0 & 50 & 0 & 0 & 50 \\
\hline B & 0 & 0 & 60 & 0 & 30 & 90 \\
\hline C & 0 & 0 & 0 & 30 & 0 & 30 \\
\hline D & 20 & 0 & 80 & 0 & 20 & 120 \\
\hline E & 0 & 0 & 90 & 10 & 0 & 100 \\
\hline TJ & 20 & 0 & 280 & 40 & 50 & 390 \\
\hline
\end{tabular}

Figure 3. An example of network cost and OD matrix

In this study, four OD matrices are required that is generated and computed:

1. OD matrix for travel cost between the origins of all passengers. (Origins-Origins)

2. OD matrix for travel cost between the destinations of all passengers. (Destinations - Destinations)

3. OD matrix for travel cost between the origins and destinations of all passengers. (Origins - Destinations)

4. OD matrix for travel cost between the taxies and origins of all passengers. (Taxies- Origins)

We need OD matrix between all origins because when a taxi gets the first passenger and start the shared travel, it goes after the second (even third or fourth) passenger, so it will be needed to calculate the cost between the origins of passengers. The structure of the OD matrix between origins is demonstrated in Figure 4.

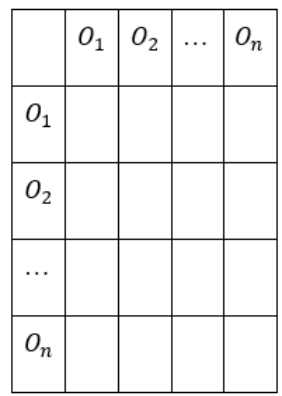

Figure 4. The structure of Origins-Origins OD matrix

In the same way, OD matrices of Destinations - Destinations, Origins - Destinations, and Taxies- Origins will be created. The structure of these matrices is displayed in Figure 5.
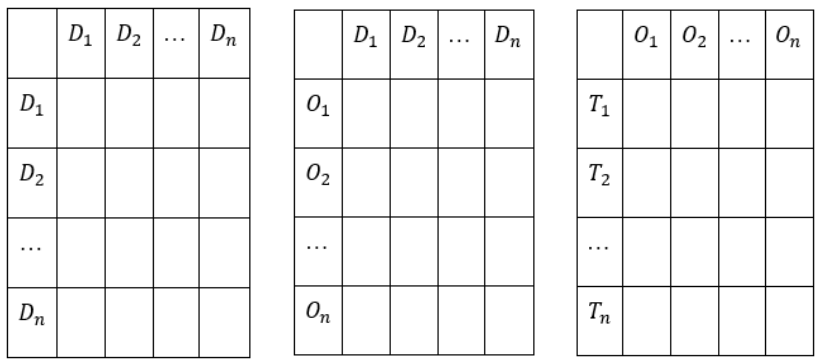

Figure 5. The structure of Destinations - Destinations, Origins Destinations and Taxies- Origins OD matrix 
The fitness function uses these OD matrices to calculate the cost of each shared taxi (length of travels), and it will be done for all taxies. Hence, the cost of all taxies will be calculated, and the total length of an answer (a chromosome) will be determined. After the initial population creation, at each stage, some of the best answers are transmitted from the present generation to the next generation by using the "selection" operator based on Roulette Wheel method (Lipowski and Lipowska, 2012). In the Roulette wheel selection method, each chromosome is assigned a weight based on the following relationship:

$$
W_{i}=\sum_{j=1}^{i} \frac{f_{i}}{f}
$$

In the above equation $f$ is the sum of the values of the fitness function of all the chromosomes. Then, a random number is selected between zero and one, and the first chromosome which its fitness function surpasses this value is selected. Naturally, chromosomes with a greater fitness function are more likely to be selected.

Secondly, several new answers are also obtained using the "crossover" operator, resulting from the combination of several rows of a matrix (chromosome) with some rows of another matrix. Thirdly, some new answers are generated by using the "mutation" operator obtained by changing a row or a passenger from one chromosome. Finally, several new randomized answers are created in the same random method to generate the initial ones, so that the next generation is created and the fitness function of all the answers is calculated again and sorted according to the lowest cost. This process continues so that the genetic algorithm converges and the best arrangement of shared passengers is specified. This is the final answer. Figure 6 depicts the flowchart of the GA algorithm.

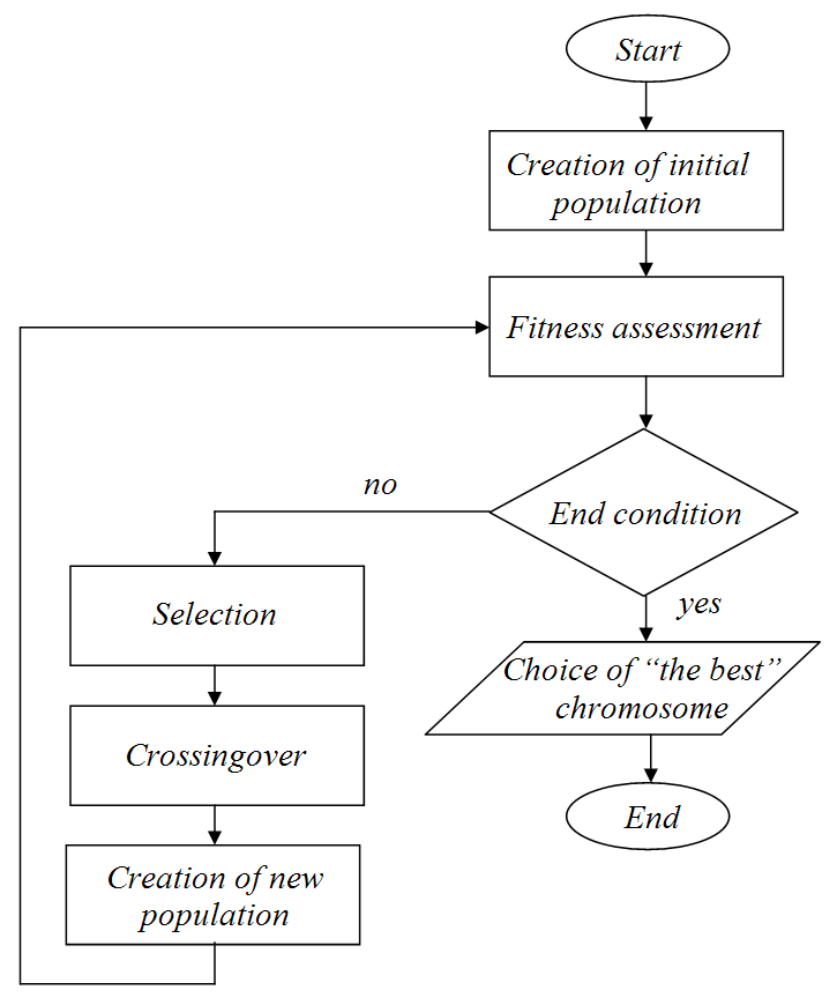

Figure 6. The GA algorithm flowchart (Whitley, 1994)

\section{RESULTS}

To evaluate the proposed model, it is implemented on a real network. The street network of region 6,7, 11 and 12 of Tehran city (the capital of Iran) are selected as a case study (Figure 7).

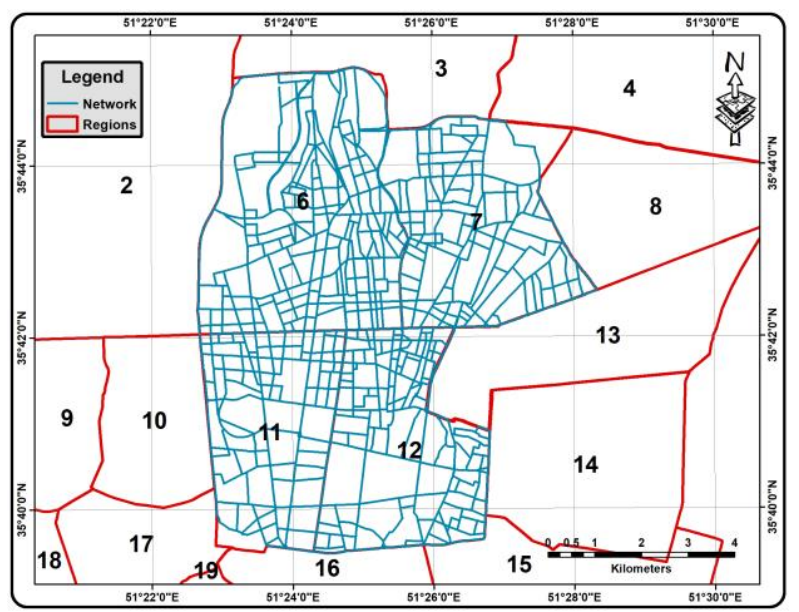

Figure 7. The study area

Then, 500 passengers are produced with random origins, destinations, request time, and maximum waiting time for ridesharing. Last two factors are used to check whether some passengers can be in a taxi logically or not. The origins and destinations are displayed in Figure 8.

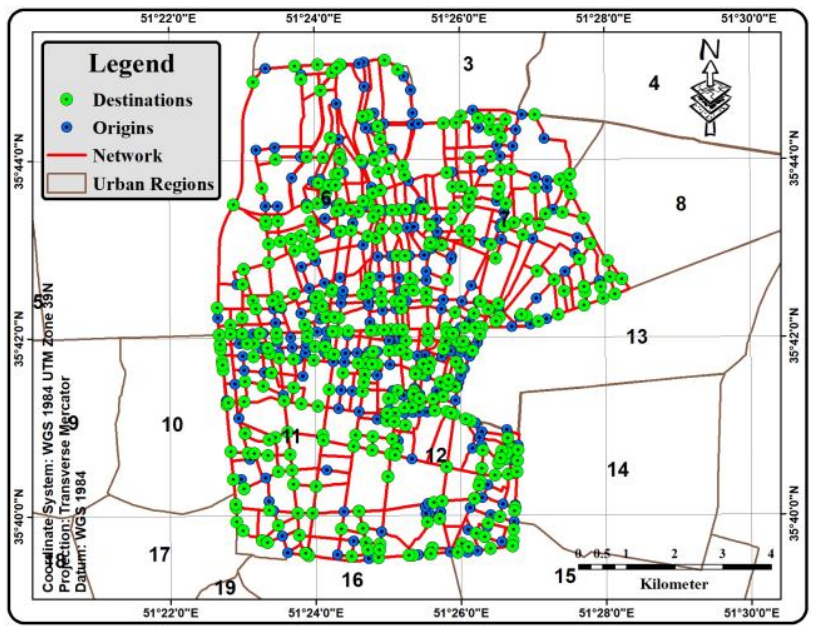

Figure 8 . The origins and destinations of passengers

Overall, the GA is processed 100 times, and the best answer (arrangements of taxies and passengers) is found. The results are presented in Table 1 .

\begin{tabular}{|l|c|c|c|}
\hline Type of taxi & number & percent & Number of passengers \\
\hline Turned off & 349 & $69.8 \%$ & $0(0 \%)$ \\
With 1 passenger & 12 & $2.4 \%$ & $12(2.4 \%)$ \\
With 2 passenger & 25 & $5 \%$ & $50(10 \%)$ \\
With 3 passenger & 18 & $3.6 \%$ & $54(10.8 \%)$ \\
With 4 passenger & 96 & $19.2 \%$ & $384(76.8 \%)$ \\
\hline
\end{tabular}

Table 1. Percentage of taxies with different number of passengers 


\section{CONCLUSION}

Results show that 349 of 500 taxies do not move in the network in comparison to the scenario when each passenger uses a taxi (or personal car), and nobody shares his ride. In this scenario, $69.8 \%$ of taxies are turned off, and this greatly helps our environment and decreases either air pollution or traffic. Besides, 25 taxies get 2 passengers, 18 taxies get 3 passengers, and 96 taxies get 4 passengers. Therefore, this means 488 passengers shared taxies, and it is equal to $97.6 \%$ drop in single-passenger taxies that has a huge impact on traffic jams. Thus, the results depict the performance of the proposed model and the customized genetic algorithm in traffic management.

\section{REFERENCES}

Böckmann, M., 2013. The Shared Economy: It is time to start caring about sharing; value creating factors in the shared economy. University of Twente, Faculty of Management and Governance.

Charles, K.K. and Kline, P., 2006. Relational costs and the production of social capital: evidence from carpooling. The Economic Journal, 116(511), pp.581-604.

Davoodi, M. and Mesgari, M.S., 2015. GIS-based route finding using ant colony optimization and urban traffic data from different sources. International Archives of the Photogrammetry, Remote Sensing and Spatial Information Sciences, 1, p.W5.

Dijkstra, E.W., 1959. A note on two problems in connexion with graphs. Numerische mathematik, 1(1), pp.269-271.

Huang, C., Zhang, D., Si, Y.W. and Leung, S.C., 2016. Tabu search for the real-world carpooling problem. Journal of Combinatorial Optimization, 32(2), pp.492-512.

Huang, S.C., Jiau, M.K. and Liu, Y.P., 2018. An ant pathoriented carpooling allocation approach to optimize the carpool service problem with time windows. IEEE Systems Journal, 13(1), pp.994-1005.

Lipowski, A. and Lipowska, D., 2012. Roulette-wheel selection via stochastic acceptance. Physica A: Statistical Mechanics and its Applications, 391(6), pp.2193-2196.

Santos, D.O. and Xavier, E.C., 2015. Taxi and ride sharing: A dynamic dial-a-ride problem with money as an incentive. Expert Systems with Applications, 42(19), pp.6728-6737.

Van Zuylen, H.J. and Willumsen, L.G., 1980. The most likely trip matrix estimated from traffic counts. Transportation Research Part B: Methodological, 14(3), pp.281-293.

Wei, X., Dai, J. and Sun, B., 2016. Routing for Taxi-pooling Problem Based on Ant Colony Optimization Algorithm.

Whitley, D., 1994. A genetic algorithm tutorial. Statistics and computing, 4(2), pp.65-85.

Zhan, X., Szeto, W.Y. and Sam, S., 2019. An Artificial Bee Colony Algorithm for the Dynamic Taxi Sharing Problem (No. 19-03116). 